[8] C. J. Anderson, "Electrical properties of an input-output cable for Josephson applications," Rev. Sci. Instrum., vol. 53, pp. 1663-1666, Nov. 1982.

[9] A. Feller, H. R. Kaupp, and J. J. DiGracomo, "Crosstalk and reflections in high-speed digital systems," in Proc. Fall Joint Computer Conf., Dec. 1965.

[10] W. T. Weeks, "Calculation of coefficients of capacitance of multiconductor transmission lines in the presence of a dielectric interface," IEEE Trans. Microwave Theory Tech., vol: MTT-18, pp. 35-43, Jan. 1970.

[11] D. A. Reitan, "Accurate determination of the capacitance of rectangular parallel-plate capacitors," Journ. Appl. Phys., vol. 30, pp. 172-176, Feb. 1959.

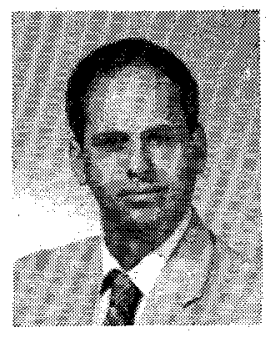

Barry J. Rubin (S'72-M'74-M'82) was born in New York, NY, in 1952. He received the B.E.E.E degree from the City College of New York, New York, in 1974.

He joined the International Business Machines Corporation in 1974 and received the M.S.E.E. degree from Syracuse University, Syracuse, New York, in 1978 and received the Ph.D. degree from the Polytechnic Institute of New York Brooklyn, in 1982. He has worked on power transistor design, CCD technology, circuit design and, since 1976, on electrical packaging analysis. He has been awarded five US patents.

\title{
Computer Analysis of Dielectric Waveguides: A Finite-Difference Method
}

\author{
EDGARD SCHWEIG, MEMBER, IEEE, AND WILLIAM B. BRIDGES, FELLOW, IEEE
}

\begin{abstract}
A method for computing the modes of dielectric guiding structures based on finite differences is described. The numerical computation program is efficient and can be applied to a wide range of problems. We report here solutions for circular and rectangular dielectric waveguides and compare our solutions with those obtained by other methods. Limitations in the commonly used approximate formulas developed by Marcatili are discussed.
\end{abstract}

\section{INTRODUCTION}

$\mathrm{D}$ IELECTRIC WAVEGUIDES of high permittivity $\left(\epsilon_{r}^{\prime} \geq 10\right)$ have been proposed as practical waveguiding structures for use in millimeter-wave integrated circuits (MMIC) [1], [2]. The prospect that the dielectric material could be a high-resistivity semiconductor raises the further possibility that active devices could be fabricated directly into the transmission line. Various practical devices for millimeter-wave applications utilizing dielectric waveguides also have been suggested: directional couplers [3], balanced mixers [3], phase shifters [4], [5], scanning antennas [6], channel-dropping filters [7]. The theoretical analysis of these devices has been based, in the case of rectangular guides, on the analytical solutions proposed by Marcatili [8], which can be expressed in simple closed forms. However, Marcatili's quasi-plane-wave analysis is based on

Manuscript received July 7, 1983; revised December 15, 1983. This work was supported by the Office of Naval Research under Contract N00014-79-C-0-0839.

The authors are with the California Institute of Technology, Department of Electrical Engineering, Pasadena, CA 91125. assumptions that are not met when the permittivity of the guide is high compared to the outer medium.

Several authors have proposed methods for the study of rectangular guides: Knox et al. [1] (modification of Marcatili's analysis), Goell [9] (expansion in circular harmonics), Schlosser [10] and Solbach [11] (mode matching), and Yeh [12], [13] (finite elements). With the exception of Solbach [11], they limit their analyses to relatively small values of permittivity $\left(\epsilon_{r}^{\prime} \simeq 2.5\right)$ and they do not give the field distributions calculated by their methods.

We have developed a numerical technique based on finite differences (FD) for computing accurate dispersion characteristics and field distribution for dielectric waveguides. This method is computationally more efficient than finite elements (FE), thus allowing the use of finer meshes, a desirable feature when accurate values of the fields are required.

\section{VARIATIONAL FORMULATION}

Both the finite-elements method (FE) and the finite-difference method $(F D)$ are based on a variational principle [14], [19]. For one-dimensional problems, the two methods are equivalent [15]. This equivalence is maintained in twodimensional problems that have simple rectangular boundaries. The advantages of a variational approach are: 1) the method does not restrict the shape of the dielectric interfaces so that complicated dielectric cross-sectional profiles can be treated; 2) the procedure is numerically. stable; and 3) it permits the use of a graded mesh that can 
be made finer in regions of particular interest or importance.

Consider a waveguide uniform in the $z$ direction which consists of isotropic, lossless dielectric media. Assume that the cross section can be divided into several subregions over which the relative permittivity is constant. Further, assume propagation along the $z$-axis of the form $\exp [j(\omega t-\beta z)]$ with longitudinal field components $H_{z}$ and $E_{z}$. In the subregion $S_{p}, H_{z}$ and $E_{z}$ satisfy the wave equations

$$
\left(\nabla_{t}^{2}+k_{p}^{2}\right)\left\{\begin{array}{c}
\phi \\
\psi
\end{array}\right\}=0
$$

where

$$
\begin{aligned}
k_{p}^{2} & \equiv K_{p} k_{0}^{2}-\beta^{2} \\
k_{0} & \equiv \frac{\omega}{c} \\
\phi & \equiv H_{z} \\
\psi & \equiv \frac{\omega \epsilon_{0}}{\beta} E_{z}
\end{aligned}
$$

and $\nabla_{t}^{2}$ is the transverse Laplacian, $c$ is the speed of light in free space, $K_{p}$ is the relative permittivity of the $p$ th subregion, and $\epsilon_{0}$ is the permittivity of space. We introduce a propagation constant normalized to free space

$$
\bar{\beta} \equiv \beta / k_{0}
$$

and a parameter $\tau_{p}$ that quantifies the discontinuity between regions $S_{P}$ and the outer medium

$$
\tau_{p} \equiv \frac{\bar{\beta}^{2}-1}{\bar{\beta}^{2}-K_{p}}
$$

The outer medium is assumed here to be free space $\left(K_{A}=1\right)$ and will be referred to by the subscript " $A$ ".

It has been shown [12], [14], [16] that the following variational expression for $k_{A}^{2}$ can be written:

$$
\begin{array}{r}
\delta J=\delta\left\{\sum_{p}\left[\tau_{p} \int_{S_{p}} \phi_{p} \nabla_{t}^{2} \phi_{p} d S+\bar{\beta}^{2} \tau_{p} K_{p} \int_{S_{p}} \psi_{p} \nabla_{t}^{2} \psi_{p} d S\right]\right. \\
\left.-k_{A}^{2}\left[\sum_{p} \int_{S_{p}} \phi_{p}^{2} d S+\bar{\beta}^{2} K_{p} \int_{S_{p}} \psi_{p}^{2} d S\right]\right\}=0
\end{array}
$$

where $J$ is a quadratic expression of the field values $\phi_{j}, \psi_{j}$ at each of the $N$ mesh points. The expression for $J$ can be further simplified (by use of the divergence theorem) so that it involves only first-order derivatives [16], [17]

$$
\begin{aligned}
J= & \sum_{p} \tau_{p} \int_{S_{p}}\left|\vec{\nabla}_{t} \phi\right|^{2} d S+\bar{\beta}^{2} \tau_{p} K_{p} \int_{S_{p}}\left|\vec{\nabla}_{t} \psi\right|^{2} d S \\
& +2 \tau_{p} \bar{\beta}^{2} \int_{S_{p}}\left(\vec{\nabla}_{t} \psi x \vec{\nabla}_{t} \phi\right)_{z} d S \\
& -k_{A}^{2}\left[\int_{S_{p}}\left|\phi_{p}\right|^{2} d S+\bar{\beta}^{2} K_{p} \int_{S_{p}}\left|\psi_{p}\right|^{2} d S\right] .
\end{aligned}
$$

This last relation is the basis of our FD procedure.



Fig. 1. To apply the FD method, the dielectric guide is enclosed in a box (metalic wall boundary conditions), and the area of interest is covered by a rectangular grid. The permittivity inside each element must be uniform.

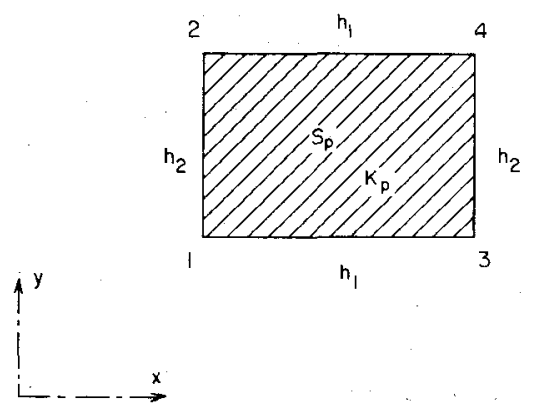

Fig. 2. A typical rectangular element $S_{\text {p }}$ used for FD approximations, of dimension $h_{1} b_{y} h_{2}$, relative permittivity $K_{p}$, and with verticies $1,2,3$, and 4 , in the order shown.

\section{FINITE-DifFERENCE RELATIONS}

To treat the problem of a dielectric guide, we first need to define a finite cross section by enclosing the guide in a "box" with electrically conducting walls sufficiently large so that it will not perturb the modes. Because of symmetry, we need to treat only one quadrant; the longitudinal electric field $E_{z}$ must be either symmetric (magnetic boundary conditions) or antisymmetric (electric boundary conditions) with respect to the $x$ - and $y$-axes, while the longitudinal magnetic field $H_{z}$ has the opposite symmetry. We then define a mesh that covers the region of interest with rectangular elements (Fig. 1). These elements are chosen so that the permittivity is constant inside each element and the electric and magnetic walls each divide a row of elements in two equal halves.

Consider one such element $S_{p}$ (Fig. 2). The contribution of this element to the variational expression $J$ can be evaluated by using FD approximations ${ }^{1}$ [17], [18]. Using $\phi_{i}$ and $\psi_{i}$ to denote the field values at point $i$, we have

$$
\begin{aligned}
\int_{S_{p}} \phi^{2} d S & \simeq \frac{h_{1} h_{2}}{4}\left(\phi_{1}^{2}+\phi_{2}^{2}+\phi_{3}^{2}+\phi_{4}^{2}\right) \\
\int_{S_{p}} \psi^{2} d S & \simeq \frac{h_{1} h_{2}}{4}\left(\psi_{1}^{2}+\psi_{2}^{2}+\psi_{3}^{2}+\psi_{4}^{2}\right) \\
\int_{S_{p}}\left|\vec{\nabla}_{t} \phi\right|^{2} d S & =\int_{0}^{h_{2}} d y \int_{0}^{h_{1}}\left(\frac{\partial \phi}{\partial x}\right)^{2} d x+\int_{0}^{h_{1}} d x \int_{0}^{h_{2}}\left(\frac{\partial \phi}{\partial y}\right)^{2} d y .
\end{aligned}
$$

\footnotetext{
${ }^{1}$ The grid lines are aligned parallel to the $x$-, $y$-axes. For convenience, we assume that point 1 is at the origin of the coordinates.
} 
For the first term, we assume that $\partial \phi / \partial x$ has the constant value $\left(\phi_{3}-\phi_{1}\right) / h_{1}$ on the segment $1-3$ and the constant value $\left(\phi_{4}-\phi_{2}\right) / h_{1}$ on the segment $4-2$. We then integrate with respect to $y$ and apply the trapezoidal rule

$$
\begin{aligned}
\int_{0}^{h_{2}} d y \int_{0}^{h_{1}}\left(\frac{\partial \phi}{\partial x}\right)^{2} d x & \simeq h_{1} \int_{0}^{h_{2}} d y\left(\frac{\partial \phi}{\partial x}\right)^{2} \\
& \simeq \frac{h_{1} h_{2}}{2}\left[\left(\frac{\phi_{4}-\phi_{2}}{h_{1}}\right)^{2}+\left(\frac{\phi_{3}-\phi_{1}}{h_{1}}\right)^{2}\right] .
\end{aligned}
$$

Likewise, we obtain for the second term

$$
\int_{0}^{h_{1}} d x \int_{0}^{h_{2}}\left(\frac{\partial \phi}{\partial y}\right)^{2} d y \simeq \frac{h_{1} h_{2}}{2}\left[\left(\frac{\phi_{2}-\phi_{1}}{h_{2}}\right)^{2}+\left(\frac{\phi_{4}-\phi_{3}}{h_{2}}\right)^{2}\right]
$$

so that

$$
\begin{aligned}
\int_{S_{p}}\left|\vec{\nabla}_{t} \phi\right|^{2} d S \simeq \frac{h_{1} h_{2}}{2}\left[\left(\frac{\phi_{4}-\phi_{2}}{h_{1}}\right)^{2}+\left(\frac{\phi_{3}-\phi_{1}}{h_{1}}\right)^{2}\right. \\
\left.+\left(\frac{\phi_{2}-\phi_{1}}{h_{2}}\right)^{2}+\left(\frac{\phi_{4}-\phi_{3}}{h_{2}}\right)^{2}\right] .
\end{aligned}
$$

A similar expression is obtained for the integral of $\left|\vec{\nabla}_{t} \psi\right|^{2}$. Finally, each term of the integral of $\left(\vec{\nabla}_{t} \psi \times \vec{\nabla}_{t} \phi\right)_{z}$ is evaluated as follows:

$$
\begin{aligned}
\int_{S_{p}} & \frac{\partial \phi}{\partial y} \frac{\partial \psi}{\partial x} d S \\
& =\int_{0}^{h_{2}} d y \int_{0}^{h_{1}} \frac{\partial \phi}{\partial y} \frac{\partial \psi}{\partial x} d x \\
& \simeq \int_{0}^{h_{2}} d y \frac{\partial \psi}{\partial x} \int_{0}^{h_{1}} \frac{\partial \phi}{\partial y} d x \\
& \simeq \frac{1}{2} h_{1} \int_{0}^{h_{2}} \frac{\partial \psi}{\partial x}\left[\left.\frac{\partial \phi}{\partial y}\right|_{x=h_{1}}+\left.\frac{\partial \phi}{\partial y}\right|_{x=0}\right] d y \\
& \simeq \frac{1}{2} h_{1}\left(\frac{\phi_{2}-\phi_{1}}{h_{2}}+\frac{\phi_{4}-\phi_{3}}{h_{2}}\right) \int_{0}^{h_{2}} \frac{\partial \psi}{\partial x} d y \\
& \simeq \frac{h_{1} h_{2}}{4}\left(\frac{\phi_{4}-\phi_{3}}{h_{2}}+\frac{\phi_{2}-\phi_{1}}{h_{2}}\right)\left(\frac{\psi_{4}-\psi_{2}}{h_{1}}+\frac{\psi_{3}-\psi_{1}}{h_{1}}\right)
\end{aligned}
$$

and

$$
\begin{aligned}
& \int_{S_{p}} \frac{\partial \phi}{\partial x} \frac{\partial \psi}{\partial y} d S \\
& \quad \simeq \frac{h_{1} h_{2}}{4}\left(\frac{\psi_{4}-\psi_{3}}{h_{2}}+\frac{\phi_{2}-\psi_{1}}{h_{2}}\right)\left(\frac{\phi_{4}-\phi_{2}}{h_{1}}+\frac{\phi_{3}-\phi_{1}}{h_{1}}\right) .
\end{aligned}
$$

The variational expression $J$ is then obtained as the sum of the contributions from each element $S_{p}$. The stationary property of $J$ is utilized by differentiating with respect to each of the $2 N$ variables $\phi_{J}$ and $\psi_{J}$. In this way, a set of $2 N$ linear equations is derived, which can be written in the form

$$
\boldsymbol{A} X=k_{A}^{2} \boldsymbol{B} X
$$

where $\boldsymbol{A}$ is a symmetric, banded matrix, $\boldsymbol{B}$ is a diagonal, positive-definite matrix, and $X$ is an ordered vector of the

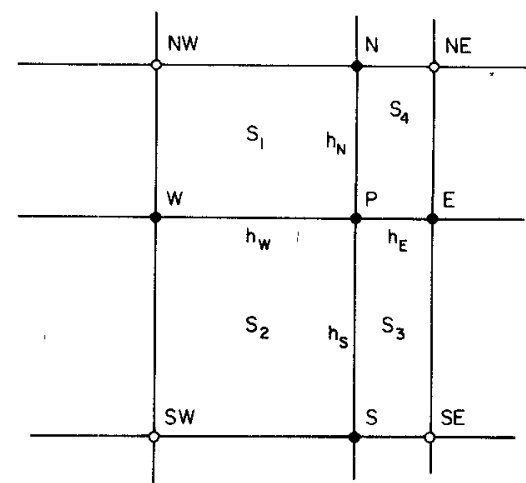

Fig. 3. Rectangular mesh. By adding the contributions of the elements $S_{1}, S_{2}, S_{3}$, and $S_{4}$, we find that the FD equations at the node $P$ are equivalent to the equations resulting from the application of the fivepoint Laplace operator for a single scalar potential.

variables $\phi_{j}, \psi_{j}$. Detailed expressions for $\boldsymbol{A}$ and $\boldsymbol{B}$ are given in the Appendix.

By a transformation, we can reduce (13) to a simple eigenvalue problem

$$
A^{\prime} X=k_{A}^{2} X
$$

and

$$
\boldsymbol{A}^{\prime}=\boldsymbol{B}^{-1 / 2} \boldsymbol{A} \boldsymbol{B}^{-1 / 2} .
$$

It is important to note that the matrix $\boldsymbol{A}^{\prime}$ is also symmetric and banded. This structure allows us to use a compact storage scheme for the numerical calculations and efficient algorithms for computing the eigenvalues and eigenvectors. In the case of the FE method [12], [13], an equation similar to (13) is obtained, but the matrix $\boldsymbol{B}$ is banded rather than diagonal, with the same bandwidth as $\boldsymbol{A}$, and therefore the eigenvalue problem is more complicated.

An estimate of the errors in this method has been made, with the result that errors in the eigenvalues will be of $O\left(h^{2}\right)$, where $h$ is the largest dimension of a rectangular element in the case of a graded mesh. This property was verified in the course of our numerical calculations on the rectangular guide by comparing the eigenvalues corresponding to successively refined grids, all other parameters remaining unchanged. The accuracy of the eigenfunctions computed numerically is much more difficult to assess, but it is usually assumed to be one order of magnitude smaller than the accuracy of the corresponding eigenvalue, i.e., $O(h)$. A detailed discussion is given in [19].

\section{Solution of the Matrix Eigenvalue EQUATIONS}

The solution of the matrix eigenvalue equation is sought in the standard form $A^{\prime} X=\lambda X$, where $A^{\prime}$ is a symmetric, banded matrix. If we use a mesh of $N \times N$ rectangular elements (Fig. 3), then the order of $A$ is $2(N-1)^{2}$ and the bandwidth (the number of subdiagonals including the main diagonal) is $2 N$. For a typical calculation $N=15$, and thus the order equals 392 and the bandwidth equals 30 .

The numerical algorithms chosen were specifically designed for a symmetric, banded matrix; the memory requirements are minimized by storing only the nontrivial 
elements. The routines employed are part of the well-known EISPACK package [20]. The solution proceeds as follows.

a) $A^{\prime}$ is reduced to tridiagonal form by a series of Givens rotations that eliminate successively each subdiagonal while maintaining the band form (routine BANDR) [20], [21].

b) The eigenvalues in an interval of interest (that is, the negative eigenvalues that are closest to zero) are determined by the routine BISECT [20], [22]. The number of eigenvalues in the given interval is computed from Sturm sequences. Next, the eigenvalues are evaluated by refining the input interval by a bisection process.

c) The eigenvectors are computed by inverse iteration (routine BANDV) [20], [23]. The system $\left(A^{\prime}-I\right) X=b$ is solved by Gaussian elimination: The right-hand-side vector $b$ is chosen to obtain a proper eigenvector.

It is important to note that by these methods it is impossible to omit any eigenvalue, which is a very desirable feature in comparison with iterative methods.

If the finite-elements formulation had been used, the resulting eigenproblem $A^{\prime} X=\lambda B X$ would involve two banded matrices. The minimum storage requirements are doubled and the numerical methods available are all iterative [12], [13], [24]

\section{Mode Designation}

All the possible modes of the rectangular guide may be divided into four classes depending on the symmetry of the longitudinal fields, since a longitudinal field $H_{z}$ of even symmetry with respect to one particular axis is always associated with a longitudinal field $E_{z}$ of odd symmetry with respect to the same coordinate. These four classes are designated in this work: $H E_{n}^{o e}, H E_{n}^{e o}, H E_{n}^{o o}, H E_{n}^{e e}$. The first superscript, $o$ or $e$, indicates the symmetry of $H_{z}$ with respect to the $x$-axis, while the second superscript denotes the symmetry of $H_{z}$ with respect to the $y$-axis. The subscript $n$ indicates the order of the given mode in its class. The correspondence between these exact modes and the designations adopted by Marcatili for his quasi-plane-wave modes is discussed later.

\section{Calculated Dispersion Curves and COMPARISON WITH OTHER WORK}

Using the FD method described above, we wrote a computer program that builds the matrices $A$ and $B$ for a guiding structure with a given distribution of relative permittivity and a given mesh. The details are given in [19]. The dielectric waveguide has a core of relative permittivity $K_{1}$ and is surrounded by an infinite medium of relative permittivity $K_{2}$. In addition to these parameters, we specify a value of $\beta / k_{o}$ (in the range $\sqrt{K_{2}}-\sqrt{K_{1}}$ for a guided mode), which in turn determines $\tau \equiv k_{2}^{2} / k_{1}^{2}$. Then the program computes the dominant eigenvalues $k_{2}^{2}$, that is, the negative eigenvalues closest to zero. For each of these eigenvalues, the corresponding value of the free-space wave-number $k_{o}$ is determined. This computation is repeated for a set of values of $\beta / k_{o}$ to obtain a complete dispersion curve. To obtain the fields, the program computes the corresponding eigenvectors.

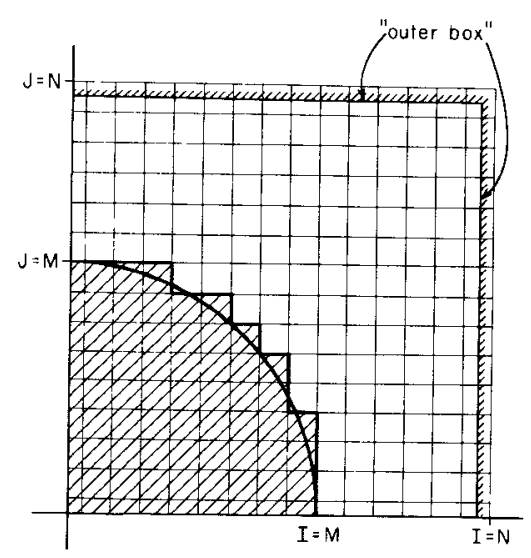

Fig. 4. Approximation of a round guide by a square mesh. The hatched elements are assigned a dielectric constant $K_{1}$, while the remaining ones correspond to $K_{2}\left(<K_{1}\right)$.

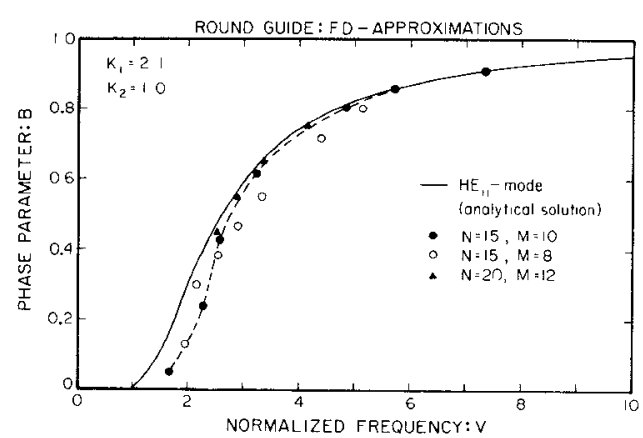

Fig. 5. Dispersion characteristic for the lowest order mode of a round waveguide: the continuous line corresponds to the exact theoretical solution for the $H E_{11}$-mode, while the dashed line is obtained from the FD method. The theoretical solution has no cutoff, and it blends smoothly with the $V$-axis to $V=0$.

The dispersion curves are presented in the $B-V$ description commonly used for optical waveguides (see [25], for example). $V$ is the normalized frequency, defined by

$$
V \equiv k_{o} a \sqrt{K_{1}-K_{2}}
$$

for a waveguide, of dimensions $a \times b$, and $B$ is the normalized phase parameter

$$
B \equiv \frac{\left(\beta / k_{o}\right)^{2}-K_{2}}{K_{1}-K_{2}} .
$$

Our results using this finite-difference method are compared below for three cases: a) the exact solution for a dielectric waveguide of circular cross section, b) the mode-matching solution of Solbach [11] for the rectangular guide, and c) the quasi-plane-wave mode solution of Marcatili [8] for a square guide.

\section{A. Round Guide}

A comparison with the round dielectric guide is particularly indicated because it is the only case for which the exact solution is known. We defined the permittivity of the elements of a square mesh in such a way that they approximate one quadrant of a circular fiber. In Fig. 4, the hatched squares are assigned a permittivity $K_{1}$ while the 




Fig. 6. Comparison between the FD and the mode-matching solutions. The mode nomenclature for the mode-matching solution is in accordance with Marcatili's. The mode nomenclature for the FD calculations is derived from the symmetry properties of the longitudinal magnetic field with respect to the $x$ - and $y$-axes. $R$ is the aspect ratio $a / b$.

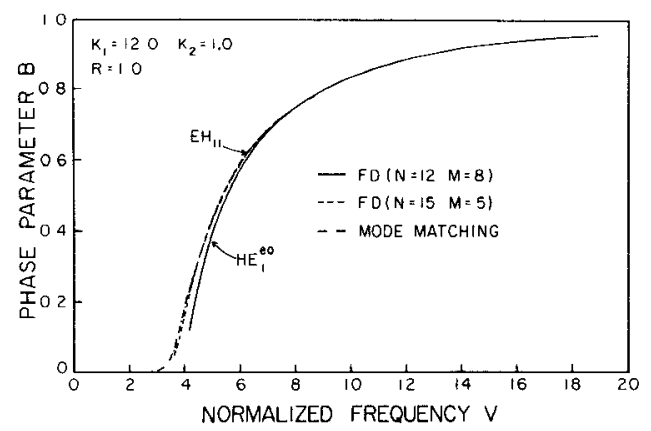

Fig. 7. Comparison among the $\mathrm{FD}$ and the mode-matching solutions for a higher relative permittivity, $K_{1}=12$.

other elements correspond to $K_{2}$. The exact dispersion curve for the dominant $\mathrm{HE}_{11}$-mode is presented in Fig. 5 along with the FD calculation. The total number of elements is $N^{2}$ while the parameter $M$ defines the number of elements in the circular section (see Fig. 4). If $M$ is chosen too small, the round guide is poorly approximated. For $N=15$ and $M=10$, the FD calculations are in very close agreement with the theoretical curve, for larger values of $B$ where the mode is well confined. For smaller values of $B$, the fields extend, further outside the dielectric, and the mode is influenced by the outer box (metallic walls). To obtain the dispersion curve in this frequency region, we need to increase the ratio $N / M$. As a benchmark, we note that for $N=15$, the CPU time required on an IBM 3032 to compute the first five eigenvalues corresponding to one value of $B$ is about $1.4 \mathrm{~min}$. For $N=20$, this time increases to approximately $4 \mathrm{~min}$.

\section{B. Mode Matching}

Mode matching has been used to compute the modes of high-permittivity image lines [11]. We compared the dispersion characteristics obtained by this method to our FD calculations in Figs. 6 and 7. The mode designations used by Solbach [11] are in accordance with those given by Marcatili [8]. The modes are designated $E H_{p q}$ when the longitudinal field is mainly electric and $H E_{p q}$ when the longitudinal field is mainly magnetic. The indices indicate the number of maxima of the dominant electric-field components. We computed by FD the modes belonging to the

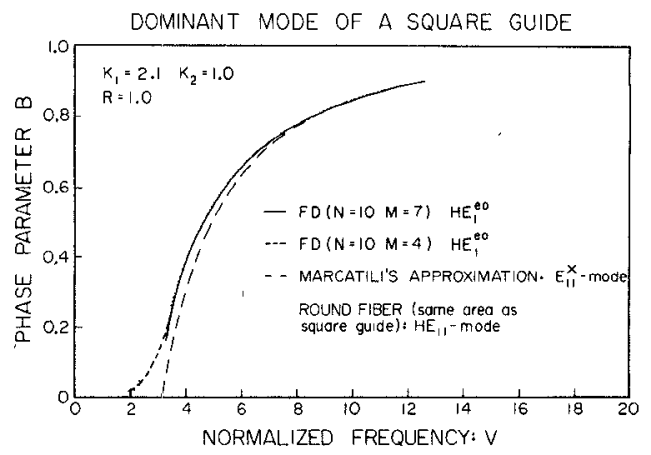

Fig. 8. Comparison among various solutions for the lowest order mode of a square dielectric guide. For obtaining an accurate FD solution at low frequencies, it is necessary to use a higher ratio $N / M$. The solution for a round fiber of equivalent cross-sectional area is shown for comparison.

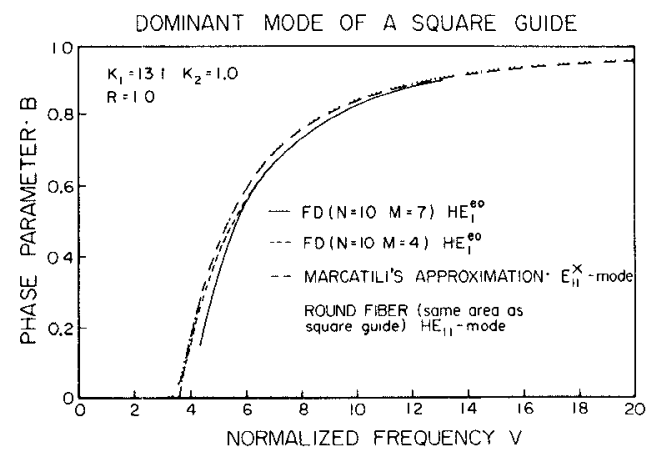

Fig. 9. Comparison among various solutions for the lowest order mode of a high-permittivity square dielectric guide. The solution for a round fiber of equivalent cross-sectional area is shown for comparison

same class of symmetry. For a permittivity of 2.22 (Fig. 6), the two techniques give slightly different values for the first mode ${ }^{2}$ but these are in closer agreement at higher values of $V$ for the second mode. For $K_{1}=12.0$, the agreement between the two methods is excellent for the lowest mode, as shown in Fig. 7.

\section{Marcatili's Approximation}

In Figs. 8 and 9, we compare the dispersion curves for the lowest order mode of a square guide of permittivity $K_{1}=2.1$ using three different methods: 1) Marcatili's quasi-plane-wave solutions, 2) the exact solution of the $H E_{11}$-mode of a round fiber that has the same area as the square guide, and 3) the FD method $\left(N^{2}\right.$ is the total number of elements and $M^{2}$ is the number of elements defining the dielectric guide).

The quasi-plane-wave modes are designated $E_{p q}^{y}$ and $E_{p q}^{x}$. The superscript indicates the direction of polarization of the dominant electric-field component while the subscript denotes the number of maxima, respectively, in the $x$ - and $y$-directions. Table I lists the symmetry properties of the longitudinal magnetic field $H_{z}$ for the four lowest order $E_{p q^{-}}^{x}$ and $E_{p q^{y}}^{y}$-modes. These properties determine the corre-

\footnotetext{
${ }^{2}$ The relative difference in $\beta / k_{o}$ is of the order of 4 percent, which is difficult to measure experimentally.
} 
TABLE I

SYMMETRY PROPERTIES OF THE FOUR LOWEST ORDER $E_{p q^{-}}^{x}$ AND $E_{p q}^{v}$-MODES

\begin{tabular}{|c|c|c|c|c|c|c|}
\hline \multirow[t]{2}{*}{ Mode } & \multicolumn{2}{|c|}{$E_{x}$} & \multicolumn{2}{|c|}{$z_{y}$} & \multicolumn{2}{|c|}{$a_{x}$} \\
\hline & $x-\operatorname{axp} 1 \mathrm{~s}$ & $y-8 \times 18$ & $x-2 \times 18$ & $y$-axis & $x-8 \times 18$ & $y-\operatorname{sx} 18$ \\
\hline $\mathbf{z}_{11}^{\mathbf{x}}$ & - & - & - & - & e & - \\
\hline $\mathbf{E}_{21}^{\mathbf{x}}$ & 。 & - & - & - & 。 & 。 \\
\hline $\mathbf{E}_{12}^{\mathbf{x}}$ & e & 。 & - & - & e & e \\
\hline $\mathbf{E}_{22}^{\mathbf{x}}$ & 。 & 。 & - & - & 。 & e \\
\hline $\mathbf{z}_{11}^{\mathbf{y}}$ & - & - & e & e & 。 & e \\
\hline $\mathbf{x}_{21}^{\mathbf{y}}$ & - & - & . & e & e & e \\
\hline $\mathrm{E}_{12}^{y}$ & - & - & e & 。 & 。 & 。 \\
\hline $\mathrm{E}_{22}^{\mathrm{y}}$ & - & - & - & . & e & 。 \\
\hline
\end{tabular}

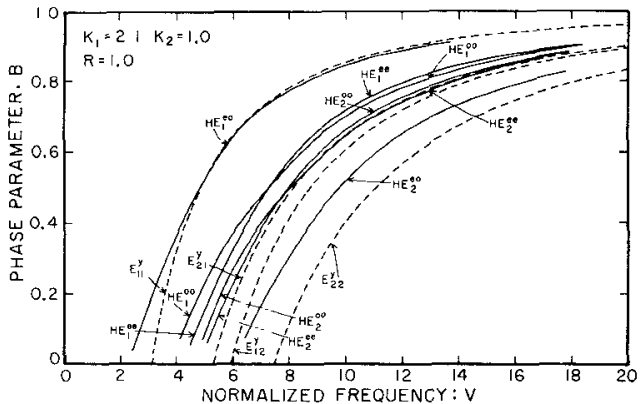

Fig. 10. Dispersion curves for a square guide. The continuous lines correspond to the FD solutions, while the dashed lines are Marcatili's.

spondence between the quasi-plane-wave mode designation and the more general mode designation used in this work.

The $B-V$ curves are compared in Fig. 9 for $K_{1}=13.1$. At high frequencies, $V \gg 1$, most of the field energy lies inside the guide, and the dispersion curves computed by 1) and 2) cannot be distinguished. This gives us a very good check on the accuracy of our FD calculations: they agree very well. Because the fields are well confined, the metalic walls can be relatively close to the guide. For small values of $V$, we know that Marcatili's solution is not correct, since it erroneously predicts a cutoff frequency greater than zero. In this region, the fields extend far outside the guide, and we expect that the dispersion curve of the square guide will be very similar to the curve of the round guide. This is confirmed by our FD calculations. In this frequency range, we needed to remove the metalic walls farther from the guide, i.e., increase the ratio $N / M$. However, for the dominant mode, the fields are expected to vary slowly inside the guide, so we can achieve the increase in $N / M$ by decreasing $M$. When the ratio $N / M$ is too small for the frequency range studied, the dispersion curve appears to drop more rapidly than it should.

For the same two values of permittivity, we have also computed $B-V$ curves for the higher order modes (Figs. 10

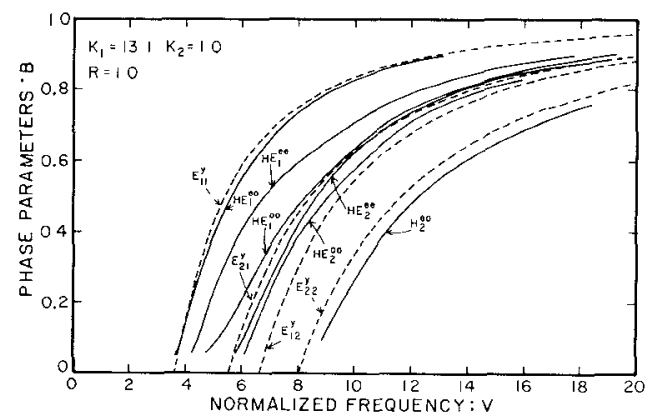

Fig. 11. Dispersion curve for a square guide. The continuous lines are the FD solutions and the dashed lines are Marcatili's.

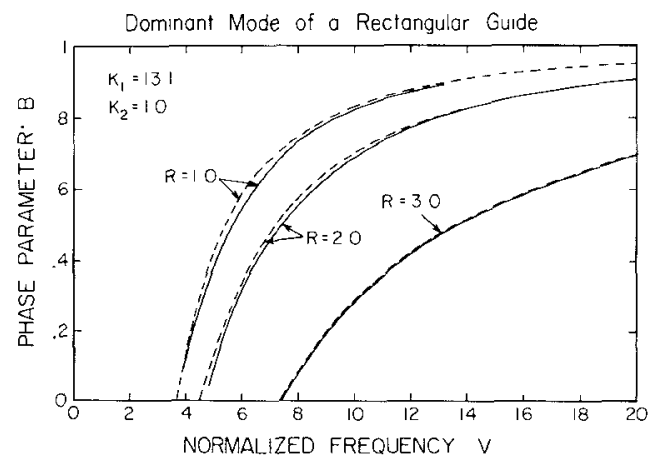

Fig. 12. Dispersion curves for rectangular high-permittivity waveguides. $R$ is the aspect ratio $a / b$ for the rectangular cross section. As the index ratio is increased, Marcatili's solution (dashed lines) becomes a better approximation, as verified by our FD calculations (continuous lines).

and 11) and compare them with Marcatili's modes that have the same axis symmetries. Because the particular waveguide that we considered is square, the $E_{p q^{-}}^{y}$ and $E_{q p}^{x}$-modes are degenerate. In the case of FD modes, the $H E_{n}^{e o}$-modes are degenerate with the $H E_{n}^{o e}$-modes.

In Figs. 10 and 11, we observe that only the dominant $E_{11}^{y}$-mode agrees well with our FD computation. The disagreement becomes progressively worse for higher order modes. We recall that the propagation constants are the stationary values of a functional (15). Therefore, for the dominant mode, deriving the eigenvalues from an assumed form for the eigenfunction, inherent in Marcatili's methods still leads to very good values for the propagation parameter. We note that the modes closest to Marcatili's $E_{21}^{y}$ - and $E_{12}^{x}$-modes are not degenerate in our FD calculations. Corresponding to each of these modes, we obtain two modes, $H E_{n}^{o o}$ and $H E_{n}^{e e}$ (with $n$ equal to 1 and 2, respectively), that become degenerate only for very large values of $V$; for a very tightly confined mode, the fields corresponding to these two modes are not much influenced by the outer dielectric interface, and the fields can be superimposed by a $45^{\circ}$ rotation.

We expect that Marcatili's solutions will become more accurate as the guide aspect ratio $R=a / b$ increases. This is confirmed by Fig. 12 that indicates that for $R=5$ we cannot distinguish the FD solution from Marcatili's.

\section{Calculated Fields}

From the preceding section, it appears that Marcatili's solutions are relatively good for the propagation constants 

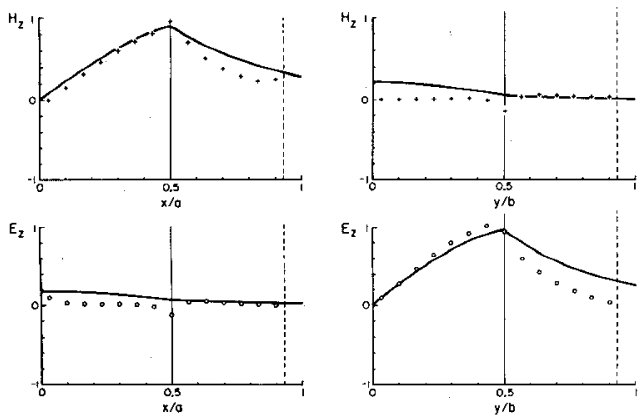

Fig. 13. Plots of the longitudinal fields of the lowest order mode of a square dielectric guide of permittivity $K_{1}=2.1\left(K_{2}=1.0\right)$. The continuous lines are the fields computed by using Marcatili's solution, while the discrete points are obtained by the FD calculations. The values of $V$ and $B$ are indicated in Table II. The fields are plotted, respectively, along lines $y=h / 2$ and $x=h / 2$, where $h$ is the computational mesh size. These are the grid lines closest to the $x$ - and $y$-axes. The square dielectric is located in the region $-0.5<x / a<+0.5,-0.5<y / b<$ +0.5 , with $a=b$. The thin dashed line indicates the position of the outer "box".
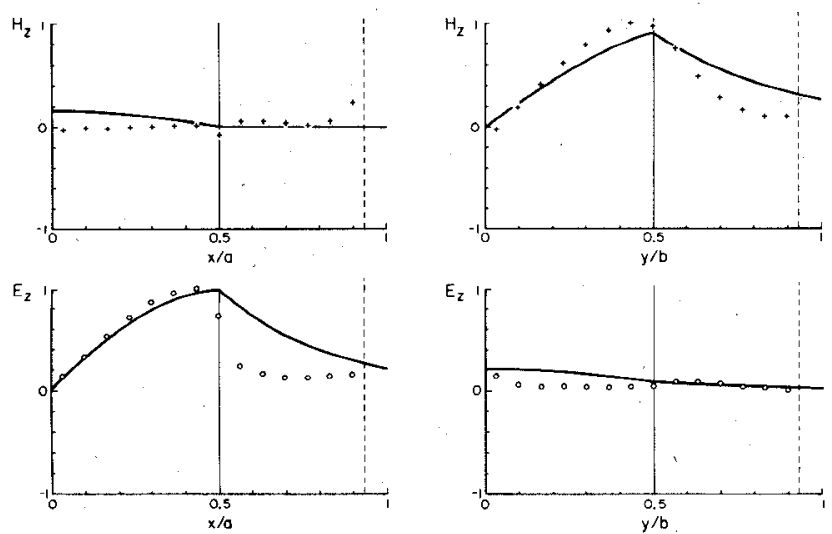

Fig. 14. Same as Fig. 13, except permittivity $K_{1}=13.1\left(K_{2}=1.0\right)$.

of the low-order mode of rectangular dielectric guides. We now compare the field behavior of the low-order modes computed by both methods.

In Fig. 13, we show the longitudinal electric and magnetic fields of Marcatili's $E_{11}^{y}$-mode, computed for square guide of permittivity $K_{1}=2.1$. The discrete points are the field values at the node points for the corresponding $H E_{1}^{o e}$-mode. The values are given along the grid line closest to the $x$ - and $y$-axes, $h / 2$ away (see Fig. 1). In Fig. 14, we compare the $E_{11}^{x}$-mode with the FD equivalent, the $H E_{1}^{e o}$ mode, for a square guide of permittivity $K_{1}=13.1$. In each case, the fields have been separately normalized so that $\left|H_{z}\right|_{\max }=1$ and $\left|E_{z}\right|_{\max }=1$. The relative importance of the $\mathrm{E}$ and $\mathrm{H}$ fields is obtained by considering, for both methods, the value of a parameter $\rho$ that indicates the dominance of $E_{\mathrm{z}}$ and $H_{\mathrm{z}}$, or vice versa

$$
\rho=\frac{\left|E_{z}\right|_{\max }}{\left|H_{z}\right|_{\max }}\left[\frac{Z_{0}}{\bar{\beta}}\right]^{-1}
$$

The quantity $\left[Z_{o} / \bar{\beta}\right]$ is the impedance of a plane wave propagating in a medium of apparent index equal to the normalized propagation constant $\bar{\beta}$. These quantities are listed in Table II. For both values of the relative permittiv-
TABLE II

COMPARISON BETWEEN MARCATILI'S SOLUTIONS AND FD SOLUTIONS ( $N=15, M=18$ ) FOR THE DOMINANT MODE OF A DIELECTRIC GUIDE

\begin{tabular}{|c|c|c|c|c|}
\hline & Mode & $\nabla$ & $B$ & $D$ \\
\hline \multirow{2}{*}{$\begin{array}{l}x_{1}=2.1 \\
x_{2}=3.0\end{array}$} & $\mathbf{z}_{11}^{x}$ & 3.44 & 0.58 & 0.91 \\
\hline & $\mathrm{mE}_{1}^{\mathrm{eo}}$ & 5.44 & 0.60 & 1.65 \\
\hline \multirow{2}{*}{$\begin{array}{l}x_{1}=: 13.1 \\
x_{2}=1.0\end{array}$} & $E_{11}^{y}$ & 6.26 & 0.62 & 16.9 \\
\hline & $\mathrm{HE}_{1}^{\mathrm{oe}}$ & 6.26 & 0.60 & 13.5 \\
\hline
\end{tabular}

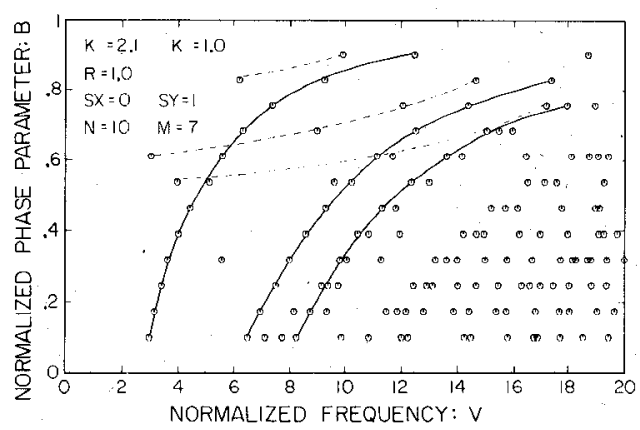

Fig. 15. Typical plot of the eigenvalues obtained by FD calculations. The continuous lines correspond to propagating modes, while the dashed lines correspond to spurious solutions. They are found to exist mostly in the range $0.5<B<1.0$.

ity, the qualitative behavior predicted by Marcatili's approximation is observed. The fields behave sinusoidally inside the waveguide and decay exponentially away from the surface. However, our FD calculations show that the fields are actually more tightly confined. In the case of the low permittivity guide $\left(K_{1}=2.1\right)$, the dominance of the $E_{z}$ over the $H_{z}$-field is underestimated by about 80 percent. There does not appear to be a clear trend in the comparison of the values of $\rho$, computed by the two methods.

\section{SPURIOUS Modes}

As explained in Section VI, the dispersion characteristics are obtained by scanning a range of values for the normalized phase parameter $B$ and computing the corresponding values of the normalized frequency $V$ for a given class of modes. The computer then generates a plot such as the one illustrated in Fig. 15. The open circles correspond each to an eigenvalue. By taking a sufficient number of values for $B$, it is possible to connect the results by smooth curves. However, this cannot be done uniquely, as shown by the two sets of lines in Fig. 15. The continuous lines correspond to the dispersion characteristics we have shown, for example, in Fig. 10. The interrupted lines do not seem to correspond to physical modes. This is verified by plotting the fields for the various modes. The "nonphysical" modes do not exhibit the simple sinusoidal/exponential behavior. Such spurious modes also have been reported by Corr and Davies [17] and Ikeuchi et al. [26]. According to Corr and 
Davies [17], these modes appear only for values of $\beta / k$ in the range

$$
K_{1} / K_{2}<\beta / k_{o}<\frac{K_{1}+K_{2}}{2 K_{2}} .
$$

For $K_{1} \gg 1$, this means that the spurious modes should be restricted to the range

$$
0.5<B<1
$$

which is generally the case in Fig. 15. However, we find a few points outside this range that do not appear to fall on curves for physically meaningful modes. We do not understand the origin of these extra points. These nonphysical solutions may arise from the indefinite nature of the variational formulation of the problem. Recall that we are computing the eigenvalues $k_{2}^{2}$ corresponding to a given (negative) value of $\tau=k_{2}^{2} / k_{1}^{2}$. For propagating modes, both $k_{1}^{2}$ and $k_{2}^{2}$ are real quantities: $k_{1}^{2}>0$ and $k_{2}^{2}<0$. A negative value of $\tau$ can also be obtained if $k_{1}^{2}$ is negative and $k_{2}^{2}$ positive. This would be the case for transient modes, that is, modes that have an imaginary value for both the propagation constant $\beta$ and the free-space wavenumber $k_{o}$.

\section{CONCLUSIONS}

We have developed a numerical method based on finitedifference (FD) for computing the propagation constants and fields of a dielectric guiding structure. The method was tested in the case of a round guide for which a rigorous analytical solution exists and also by comparison with other work. The FD method gives good agreement, even with relatively small meshes. It is more efficient than the finite-elements method (FE) by a factor of 2 in terms of computer storage. This would allow us to treat larger meshes, being limited only by CPU time. The FD method is also more favorable than the FE method in terms of the numerical methods available for computing the eigenvalues of the resulting linear eigenproblem.

We have compared our solutions to Marcatili's quasiplane-wave solutions in the case of square guides that have a permittivity much larger than the surrounding medium. We found that Marcatili's solution compares closely in dispersion curves, but only for the lowest order mode. However, because most practical dielectric waveguides are used in a single-mode configuration, this does not diminish the usefulness of Marcatili's closed-form solution for propagation constants and approximate fields. Devices that depend critically on the details of the fields outside the dielectric region (such as directional couplers) may require the FD calculations.

Finally, the FD method can be applied to guiding structures of arbitrary shape and dielectric distribution.

\section{APPENDIX \\ FD-MATRICES}

The finite-differences (FD) method utilizes a mesh that covers the region of interest with rectangular elements in
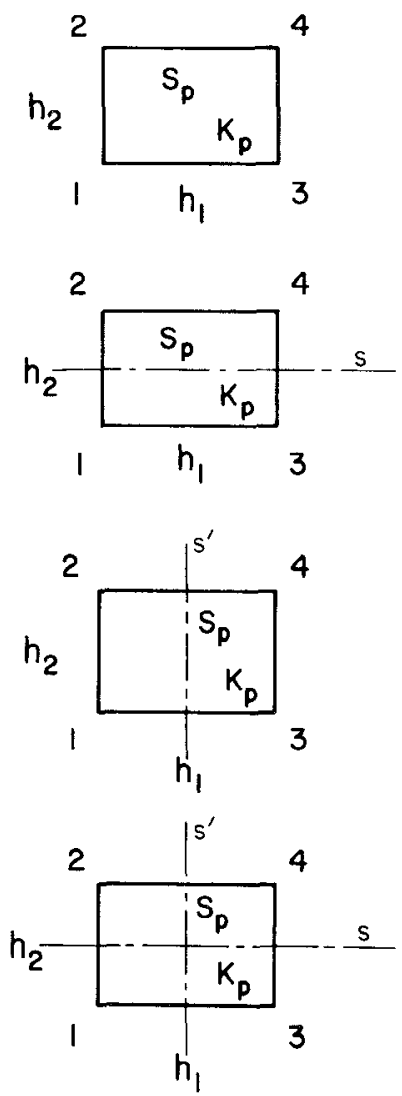

Fig. 16. The element used for FD calculations can either be (a) the general element or (b), (c) an element divided by one or (d) two symmetry axes. The parameters $s$ and $s^{\prime}$ define the symmetry of the longitudinal magnetic field with respect to the $x$ - and $y$-axis: $s$ and $s^{\prime}$ take the values \pm 1 . For example, $\left.H_{z}\right|_{1}=\left.s H_{z}\right|_{2}$, while $\left.H_{z}\right|_{1}=\left.s^{\prime} H_{z}\right|_{3}$.

such a way that the boundaries and the two axes of symmetry divide elements into two equal halves. Therefore, we have four types of rectangular elements:

a) the general rectangular element (Fig. 16(a));

b) the element divided in two by a line parallel to the $x$-axis (Fig. 16(b));

c) the element divided in two by a line parallel to the $y$-axis (Fig. 16(c));

d) the element divided by two orthogonal lines (Fig. $16(d))$.

On each of these lines, an electric or magnetic boundary condition must be applied. The quantities $s$ and $s^{\prime}$ define the symmetry of the longitudinal field $\phi=H_{z}$ with respect to one of these axes: $s$ and $s^{\prime}$ take the values \pm 1 . If, for example, for the element illustrated in Fig. 16(d), $s$ takes the value +1 , this signifies that $\phi$ is an even function about the line and, therefore, $\psi=\left(\omega \epsilon_{0} / \beta\right) E_{z}$ must be an odd function: $\phi_{2}=\phi_{1}, \phi_{4}=\phi_{3}, \psi_{2}=-\psi_{1}, \psi_{4}=-\psi_{3}$. This is equivalent to an electric boundary condition.

We can now evaluate the contribution of these elements to the FD matrices $\boldsymbol{A}$ and $\boldsymbol{B}$ by using the FD approximations. For each element $S_{p}$, we can write a relation of the form

$$
\left[\frac{\partial J_{p}}{\partial \phi_{l}}, \frac{\partial J_{p}}{\partial \psi_{l}}\right]^{T}=A_{p}\left[\phi_{l}, \psi_{l}\right]^{T}-k_{A}^{2} B_{p}\left[\phi_{l}, \psi_{l}\right]^{T}
$$


these relations are then summed to form $\boldsymbol{A} X=k_{A}^{2} \boldsymbol{B} X$. For the general element (Fig. 16(a)), (A1) becomes ${ }^{1}$

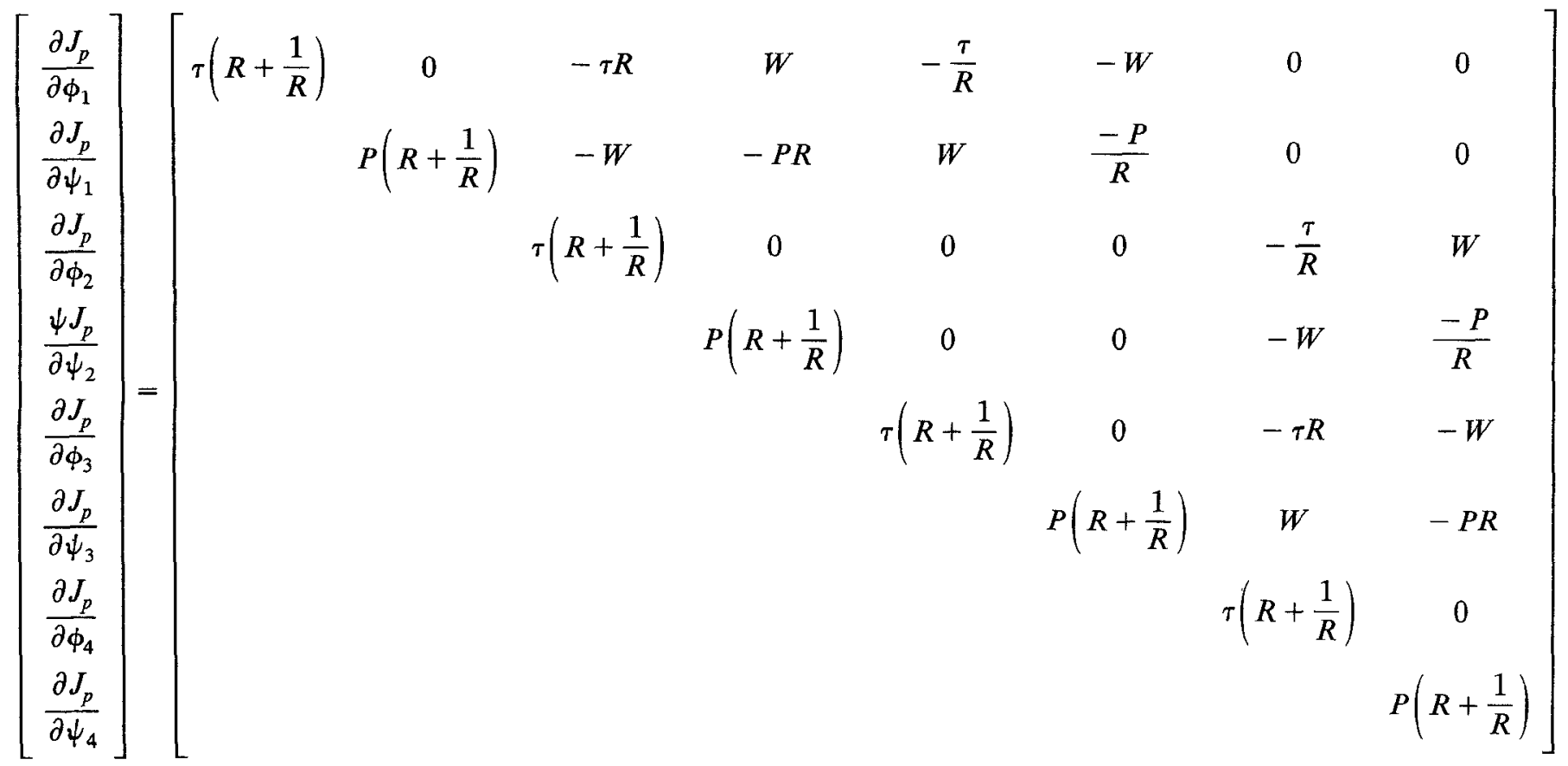$$
\left[\begin{array}{l}
\phi_{1} \\
\psi_{1} \\
\phi_{2} \\
\psi_{2} \\
\phi_{3} \\
\psi_{3} \\
\phi_{4} \\
\psi_{4}
\end{array}\right]-\frac{h_{1} h_{2}}{2} k_{A}^{2} \operatorname{diag}\left[1, P^{\prime}, 1, P^{\prime}, 1, P^{\prime}, 1, P^{\prime}\right]\left[\begin{array}{l}
\phi_{1} \\
\psi_{1} \\
\phi_{2} \\
\psi_{2} \\
\phi_{3} \\
\psi_{3} \\
\phi_{4} \\
\psi_{4}
\end{array}\right]
$$

where

$$
\begin{aligned}
\tau & =\frac{\bar{\beta}^{2}-1}{\bar{\beta}^{2}-K_{p}} \\
P^{\prime} & =\bar{\beta}^{2} K_{p} \\
P & =\bar{\beta}^{2} K_{p} \tau \\
W & =\bar{\beta}^{2} \tau .
\end{aligned}
$$


Using the symmetry parameters $s$ and $s^{\prime}$, we can compute the contribution of the element illustrated. In Fig. 16(a):

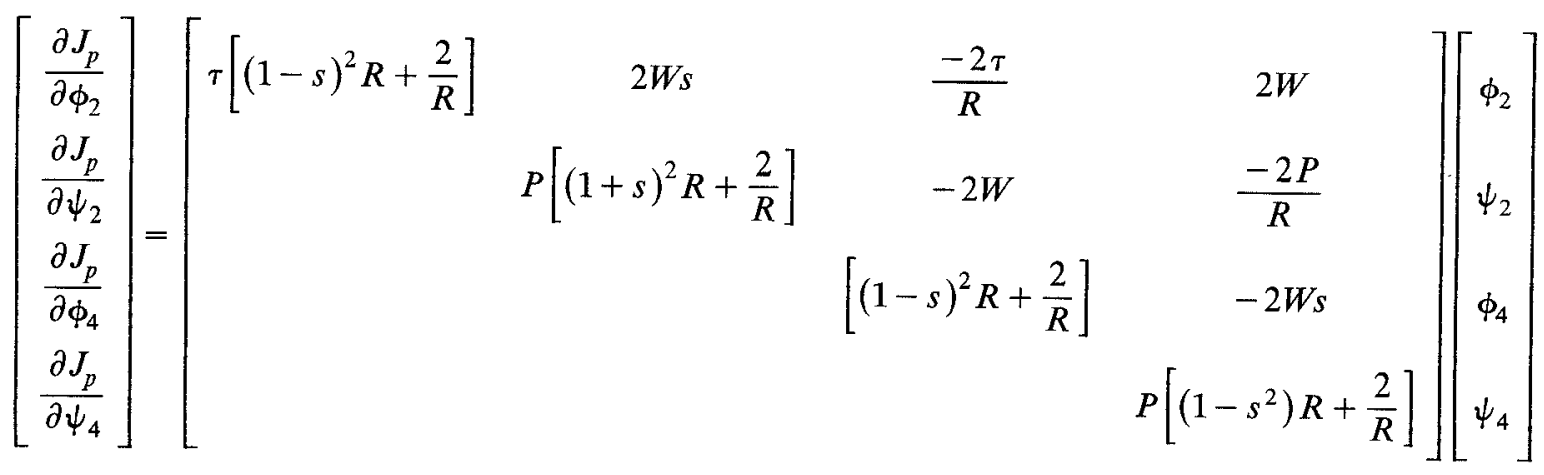

$$
-k_{A}^{2} h_{1} h_{2} \operatorname{diag}\left[1, P^{\prime}, 1, P^{\prime}\right]\left[\begin{array}{c}
\phi_{2} \\
\psi_{2} \\
\phi_{4} \\
\psi_{4}
\end{array}\right]
$$

In Fig 16(c)

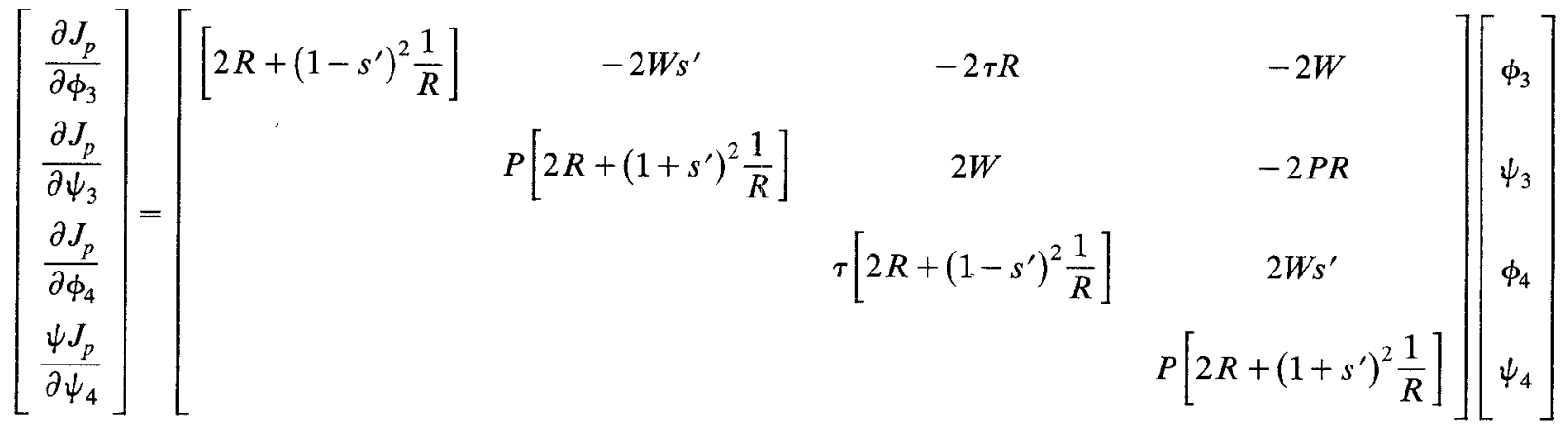

$$
\begin{aligned}
& -k_{A}^{2} h_{1} h_{2} \operatorname{diag}\left[1, P^{\prime}, 1, P^{\prime}\right]\left[\begin{array}{l}
\phi_{3} \\
\psi_{3} \\
\phi_{4} \\
\psi_{4}
\end{array}\right] .
\end{aligned}
$$

In Fig. 16(d)

$$
\left[\begin{array}{c}
\frac{\partial J_{p}}{\partial \phi_{1}} \\
\frac{\partial J_{p}}{\partial \psi_{1}}
\end{array}\right]=\left[\begin{array}{cc}
2 \tau\left[\left(1-s^{\prime}\right)^{2} \frac{1}{R}+(1 A)^{1} R\right] & -4 W\left(s^{\prime}-s\right) \\
-4 W\left(s^{\prime}-s\right) & 2 P\left[\left(1+s^{\prime}\right)^{2} \frac{1}{R}+(1+s)^{2} R\right]
\end{array}\right]\left[\begin{array}{l}
\phi_{1} \\
\psi_{1}
\end{array}\right]-k_{A}^{2} h_{1} h_{1} \operatorname{diag}\left[1, P^{\prime}\right]\left[\begin{array}{l}
\phi_{1} \\
\psi_{1}
\end{array}\right] .
$$


These relations are then used by a program to build the matrices $A$ and $B$ systematically by adding the contribution of each element numerically. The necessary inputs to the routine are:

the total number of mesh elements;

the local ${ }^{2}$ mesh size $h_{1}, h_{2}$;

the normalized propagation constant $\bar{\beta}=/ k_{0}$;

the permittivity $K_{p}$ at the location of the element $S_{p}$;

the symmetry parameters $s$ and $s^{\prime}$ that define the various modes.

\section{REFERENCES}

[1] R. M. Knox and P. P. Toulios, "Integrated circuits for the millimeter through optical frequency range," in Proc. of the Symp. Submillimeter Waves, New York, Mar. 1970, pp. 497-516.

[2] R. M. Knox, "Dielectric waveguide microwave integrated circuits-An overview," IEEE Trans. Microwave Theory Tech., vol. MTT-24, pp. 806-814, Nov. 1976.

[3] J. A. Paul and Y. W. Chang, "Millimeter-wave image-guide integrated passive devices," IEEE Trans. Microwave Theory Tech., vol. MTT-26, pp. 751-754, Oct. 1978.

[4] H. Jacobs and M. M. Chrepta, "Electronic phase shifter for millimeter-wave semiconductor dielectric integrated circuits," IEEE Trans. Microwave Theory Tech., vol. MTT-22, pp. 411-417, Apr. 1974.

[5] C. H. Lee, P. S. Mak, and A. P. DeFonzo, "Optical control of millimeter-wave propagation in dielectric waveguides," IEEE $J$. Quantum Electron., vol. QE-16, pp. 277-288, Mar. 1980.

[6] K. L. Klohn, R. E. Horn, H. Jacobs, and E. Freibergs, "Silicon waveguides frequency scanning linear array antenna," IEEE Trans. Microwave Theory Tech., vol. MTT-26, pp. 764-773, Oct. 1978.

[7] T. Itanami and S. Shindo, "Channel-dropping filter for millimeterwave integrated circuits," IEEE Trans. Microwave Theory Tech. vol. MTT-26, pp. 759-764, Oct. 1978.

[8] E. A. J. Marcatili, "Dielectric rectangular waveguide and directional coupler for integrated optics," Bell Syst. Tech. J., vol. 48, pp. 2071-2132, Sept. 1969.

[9] J. E. Goell, "A circular-harmonic computer analysis of rectangular dielectric waveguides," Bell. Syst. Tech. J., vol. 48, pp. 2133-2160, Sept. 1969.

[10] W. Schlosser and H.-G. Unger, "Partially-filled waveguides and surface waveguides of rectangular cross section," in Advances of Microwaves, vol. 1. New York: Academic Press, 1966, pp. 319-387.

[11] K. Solbach and I. Wolff, "The electromagnetic field and phase constants of dielectric image lines," IEEE Trans. Microwave Theory Tech., vol. MTT-26, pp. 266-274, Apr. 1978.

[12] C. Yeh, S. B. Dong, and W. Oliver, "Arbitrarily shaped inhomogeneous optical fiber or integrated optical waveguides," J. Appl. Phys., vol. 46, pp. 2125-2129, May 1975.

[13] C. Yeh, K. Ha, S. B. Dong, and W. P. Brown, "Single-mode optical waveguides," Appl. Opt., vol. 18, pp. 1490-1504, May 1979.

[14] A. D. Berk, "Variational principles for electromagnetic resonators and waveguides," IRE Trans. Antennas Propagat., vol. AP-4, pp. 104-111, Apr. 1956.

[15] A. R. Mitchell and D. F. Griffiths, The Finite Difference Method in Partial Differential Equations. New York: Wiley, 1980.

[16] S. Ahmed and P. Daly, "Finite-element methods for inhomogeneous waveguides," Proc. IEEE, vol. 116, pp. 1661-1664, Oct. 1969

[17] D. G. Corr and J. B. Davies, "Computer analysis of the fundamental and higher order modes in single coupled microstrip," IEEE Trans. Microwave Theory Tech., vol. MTT-20, pp. 669-678, Oct. 1972.

[18] E. Butkov, Mathematical Physics. Reading, MA: Addison-Wesley, 1968.

[19] E. Schweig, "Dielectric waveguides for millimeter waves," $\mathrm{Ph} . \mathrm{D}$. dissertation, California Institute of Technology, Pasadena, CA, 1982.

[20] B. S. Garbow, J. M. Boyle, J. J. Dougarra, and C. B. Moler, "Matrix eigensystem routines-EISKPACK guide extension," in

\footnotetext{
${ }^{2}$ When a graded mesh is used, the dimensions $h_{1}$ and $h_{2}$ of each element $S_{p}$ become a function of the position of the element.
}

Lecture Notes in Computer Science, vol. 51. Heidelberg: SpringerVerlag, 1977.

[21] H. R. Schwartz, "Tridiagonalization of a symmetric band matrix," Numer. Math., vol. 12, pp. 231-241, 1968.

[22] W. Barth, R. S. Martin, and J. H. Wilkinson, "Calculation of the eigenvalues of a symmetric tridiagonal matrix by the method of bisection," Numer. Math., vol. 9, pp. 336-393, 1967.

[23] J. H. Wilkinson, "Calculation of the eigenvectors of a symmetric tridiagonal matrix by inverse iteration," Numer. Math., vol. 4, pp. $368-376,1962$

[24] G. Peters and W. H. Wilkinson, "Eigenvalues of $A x=\lambda B x$ with band symmetric $A$ and $B$," Comput. J., pp. 398-404, 1969.

[25] H.-G. Unger, Planar Optical Waveguides and Fibers. Oxford: Clarendon Press, 1977.

[26] Masatoshi Ikeuchi, Hideo Sawami, and Hiroshi Niki, "Analysis of open-type dielectric waveguides by the finite-element iterative method," IEEE Trans. Microwave Theory Tech., vol. MTT-29, pp. 234-239, Mar. 1981.

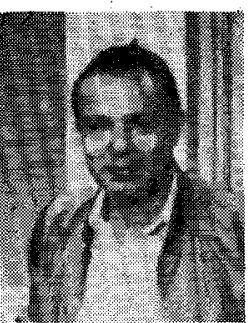

Fdgard Schweig (S'78-M'82) was born in Brussels, Belgium, on December 8, 1955. He received the "Ingénier Civil Mécanicien-Electicien" degree from the University of Brussels in 1977. He received the M.S. degree and $\mathrm{Ph} . \mathrm{D}$. degree, both in electrical engineering, from the California Institute of Technology, Pasadena, in 1978 and 1982 , respectively.

While at Caltech, he was engaged in research on dielectric waveguides for millimeter and submillimeter wave transmission, and measurement of the dielectric properties of materials in the millimeter-wave range. He also undertook studies of time-resolved optical absorption in solid-state materials with nanosecond pulsed carbon dioxide lasers. He was employed by the Peruvian national electrical utility, Electoperu, in 1982, and currently heads his own consulting firm, Milimega, in Lima, Peru.



William B. Bridges (S'53-M'61-F'70) was born in Inglewood CA, on November 29;1934. He received the B.S., M.S., and Ph.D. degrees in electrical engineering from the University of California, Berkeley, in 1956, 1957, and 1962, respectively, and was an Associate Professor in Electrical Engineering from 1957 to 1959 , teaching courses in communication and circuits. His graduate research dealt with noise in microwave tubes and electron-stream instabilities. Summer jobs at RCA and Varian provided stimulating experience with microwave radar systems, ammonia beam masers, and the early development of the ion vacuum pump.

He joined the Hughes Research Laboratories in 1960 as a member of the Technical Staff, and was a Senior Scientist from 1968 to 1977 , with a brief tour as Manager of the Laser Department in 1969-70. His research at Hughes involved gas lasers of all types and their application to optical communication, radar, and imaging systems. He is the discoverer of laser oscillation in noble gas ions and spent several years on the engineering development of practical high-power visible and ultraviolet ion lasers for military applications. He joined the faculty of the California Institute of Technology in 1977 as Professor of Electrical Engineering and Applied Physics. He served as Executive Officer for Electrical Engineering from 1979-81. In 1983, he was appointed Carl F. Braun Professor of Engineering. He currently conducts research in millimeter/submillimeter-wave technology and gas laser discharges.

Dr. Bridges is member of Eta Kappa Nu, Tau Beta Pi, Phi Beta Kappa and Sigma Xi, receiving Honorable Mention from Eta Kappa Nu as "The Outstanding Young Electrical Engineer for 1966." He is a member of the National Academy of Engineering and the National Academy of Sciences and a Fellow of the Optical Society of America. He was a Sherman Fairchild Distinguished Scholar at Caltech in 1974-75. He is co-author (with C. K. Birdsall) of Electron Dynamics of Diode Regions (New York: Academic Press, 1966). He has served on various committees of the IEEE and OSA, and was formerly Associate Editor of the IEEE Journal of Quantum Electronics and the Journal of the Optical Society of America. He currently serves on the OSA Board of Directors. 